\title{
SCATTERING OF VORTICES IN THE ABELIAN HIGGS MODEL
}

\author{
ROMAN PALVELEV
}

Communicated by Theodore Voronov

Abstract. We study the scattering of vortices in the Abelian (2+1)-dimensional Higgs model. We show that in the case of the symmetric head-on collision of $N$ vortices their trajectories are rotated by the angle $\pi / N$ after the collision.

\section{Introduction}

In this paper we study the scattering of vortices in the Abelian (2+1)-dimensional Higgs model. The vortices, we are considering, are solutions of the vortex equations, arising in the superconductivity theory. They are given by smooth pairs $(A, \Phi)$, consisting of the (electromagnetic) gauge potential $A$ and the (scalar) Higgs field $\Phi$ on $\mathbb{C}$. Such solutions are parameterized (up to gauge equivalence) by the zeros of the Higgs field $\Phi$, so the moduli space of $N$ vortices can be identified with $\mathbb{C}^{N}$. The dynamics of vortices in $\mathbb{C}$ is governed by the hyperbolic Ginzburg-Landau action functional. The dynamics of $N$ vortices may be described approximately by geodesics of $\mathbb{C}^{N}$ in the metric, determined by the kinetic energy of the model. Unfortunately, this metric cannot be computed explicitly. But in a special case of the symmetric scattering of $N$ vortices we can show, without using the explicit form of the metric, that after their head-on collision the configuration of vortices looks the same, only rotated by the angle $\pi / N$. In particular, in the case of two vortices, their trajectories are rotated by the angle $\pi / 2$ after the head-on collision, so we have the right-angle scattering. This result was already obtained earlier in a number of papers (see [1,4-6]).

\section{Vortex Solutions in the Abelian Higgs Model}

The two-dimensional Abelian Higgs model is determined by the following action functional

$$
V(\alpha, \Phi)=\frac{1}{2} \int_{\mathbb{R}^{2}}\left(\left|\mathrm{~d}_{\alpha} \Phi\right|^{2}+F_{12}^{2}+\frac{\lambda}{4}\left(|\Phi|^{2}-1\right)^{2}\right) \mathrm{d} x \mathrm{~d} y
$$


where $\mathrm{d}_{\alpha}=\mathrm{d}+\alpha, \alpha=-\mathrm{i} A_{1} \mathrm{~d} x-\mathrm{i} A_{2} \mathrm{~d} y$ is a gauge potential with smooth real-valued coefficients $A_{1}, A_{2}$ on $\mathbb{R}^{2}, \Phi=\Phi_{1}+\mathrm{i} \Phi_{2}$ is the Higgs field, given by a smooth complex-valued function on $\mathbb{R}^{2}, \lambda>0$ is a constant. We denote by $F_{12}:=\partial_{1} A_{2}-\partial_{2} A_{1}$ the gauge field, generated by $\left(A_{1}, A_{2}\right)$, and set $\partial_{1}:=\partial_{x}$, $\partial_{2}:=\partial_{y}$.

The action functional $V$ is invariant under gauge transformations of the type

$$
\alpha \longmapsto \tilde{\alpha}=\alpha-\mathrm{id} \chi, \quad \Phi \longmapsto \tilde{\Phi}=\mathrm{e}^{\mathrm{i} \chi} \Phi
$$

where $\chi$ is a smooth real-valued function on $\mathbb{R}^{2}$.

Integrating by parts, we can rewrite the action functional $V$ in the Bogomolny form

$$
\begin{aligned}
V= & \frac{1}{2} \int_{\mathbb{R}^{2}}\left\{\left(\left(\partial_{1} \Phi_{1}+A_{1} \Phi_{2}\right) \mp\left(\partial_{2} \Phi_{2}-A_{2} \Phi_{1}\right)\right)^{2}\right. \\
& \left.+\left(\left(\partial_{2} \Phi_{1}+A_{2} \Phi_{2}\right) \pm\left(\partial_{1} \Phi_{2}-A_{1} \Phi_{1}\right)\right)^{2}+\left(F_{12} \pm \frac{1}{2}\left(|\Phi|^{2}-1\right)\right)^{2}\right\} \mathrm{d} x \mathrm{~d} y \\
& \pm \frac{1}{2} \int_{\mathbb{R}^{2}} F_{12} \mathrm{~d} x \mathrm{~d} y+\frac{\lambda-1}{4} \int_{\mathbb{R}^{2}}\left(|\Phi|^{2}-1\right)^{2} \mathrm{~d} x \mathrm{~d} y .
\end{aligned}
$$

Hereafter, we consider only the critical case $\lambda=1$ (cf. [2]). Then the right hand side is the sum of non-negative terms and the topological term $\frac{1}{2} \int_{\mathbb{R}^{2}} F_{12} \mathrm{~d} x \mathrm{~d} y$, which is proportional to the topological charge or the vortex number $N$ of the field $(\alpha, \Phi)$. This is the integer number that equals (cf. [2])

$$
\frac{1}{2 \pi} \int_{\mathbb{R}^{2}} F_{12} \mathrm{~d} x \mathrm{~d} y=N
$$

if the following conditions are satisfied: $F_{12} \in L^{1}\left(\mathbb{R}^{2}\right),|\Phi| \rightarrow 1$ for $r:=$ $\sqrt{x^{2}+y^{2}} \rightarrow \infty$ and $\left|\mathrm{d}_{\alpha} \Phi\right| \leq C / r^{1+\delta}$. Evidently, $N$ does not change under gauge transformations.

Fix a vortex number $N$ and assume that $N \geq 0$. Then the Bogomolny formula implies that $V(\alpha, \Phi) \geq \pi N$ and the minimal value of $V$ (equal to $\pi N$ ) is attained on solutions of the system of equations

$$
\left\{\begin{aligned}
\partial_{1} \Phi_{1}+A_{1} \Phi_{2} & =\partial_{2} \Phi_{2}-A_{2} \Phi_{1} \\
\partial_{2} \Phi_{1}+A_{2} \Phi_{2} & =-\partial_{1} \Phi_{2}+A_{1} \Phi_{1} \\
F_{12} & =-\frac{1}{2}\left(|\Phi|^{2}-1\right)
\end{aligned}\right.
$$


called the vortex equations. These equations are invariant under gauge transformations.

Introduce the complex coordinate $z=x+\mathrm{i} y$ on the plane. The following existence and uniqueness theorem is proved in [2].

Theorem 1. (Taubes) Suppose that $N \geq 0$ and $Z_{1}, Z_{2}, \ldots, Z_{N}$ are arbitrary (not necessarily distinct) points on the complex plane. Then there exists a solution $\left(A_{1}, A_{2}, \Phi\right)$ of the vortex equations such that the zeroes of $\Phi$ coincide with $Z_{1}, \ldots, Z_{N}$ and

$$
\Phi(z, \bar{z}) \sim c_{j}\left(z-Z_{j}\right)^{n_{j}}
$$

in a neighborhood of each $Z_{j}$. Here $n_{j}$ is the multiplicity of $Z_{j}$ in the collection $\left\{Z_{1}, \ldots, Z_{N}\right\}, c_{j}$ is a nonzero constant.

For this solution $\left|\mathrm{d}_{\alpha} \Phi\right| \leq C(1-|\Phi|)$ for some $C>0$ and for any $\delta>0$ there exists a number $C(\delta)>0$ such that $1-|\Phi| \leq C(\delta) \mathrm{e}^{-(1-\delta)|z|}$.

The vortex number of this solution is equal to $N$ and the solution with the indicated properties is uniquely defined up to gauge equivalence.

The solution, whose existence is asserted in the above Theorem, is called the $N$ vortex solution.

The moduli space of $N$-vortex solutions, denoted by $\mathcal{M}_{N}$, is the set of classes of $N$-vortex solutions up to gauge equivalence. Using the Taubes theorem, we can identify it with the $N$ th symmetric power $S^{N} \mathbb{C}$, i.e., with the set of unordered collections of $N$ complex numbers (equal to zeroes of $\Phi$ ). This symmetric power may be identified with $\mathbb{C}^{N}$ by assigning to any collection $\left\{Z_{1}, \ldots, Z_{N}\right\}$ the monic polynomial $p(z)$ with zeroes at $Z_{1}, \ldots, Z_{N}$

$$
p(z)=\left(z-Z_{1}\right)\left(z-Z_{2}\right) \ldots\left(z-Z_{N}\right)=z^{N}+S_{1} z^{N-1}+\cdots+S_{N-1} z+S_{N} .
$$

\section{The Tangent Bundle of $\mathcal{M}_{N}$ and Kinetic Energy Metric on $\mathcal{M}_{N}$}

The tangent space to $\mathcal{M}_{N}$ at any point coincides with $\mathbb{C}^{N}$. However, following [7], we can give a more detailed description of the tangent bundle of $\mathcal{M}_{N}$. This description of $T \mathcal{M}_{N}$ will be used in the definition of a special kinetic energy metric on $\mathcal{M}_{N}$.

Given arbitrary points $\left\{Z_{1}, \ldots, Z_{N}\right\} \in \mathcal{M}_{N}$, we choose a particular $N$-vortex solution $\left(A_{1}, A_{2}, \Phi\right)$, associated with $\left\{Z_{1}, \ldots, Z_{N}\right\}$ by the Taubes theorem, by fixing the gauge in the following way:

$$
\Phi(z)=\left(z-Z_{1}\right)\left(z-Z_{2}\right) \ldots\left(z-Z_{N}\right) f(z) \text { with } f(z)>0 .
$$


We consider now the linearized vortex equations (at the solution $\left(A_{1}, A_{2}, \Phi\right)$ ), having the form

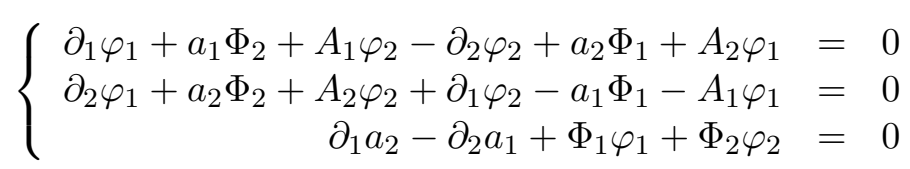

where $\left(a_{1}, a_{2}, \varphi\right)$ is the perturbation, $\varphi=\varphi_{1}+\mathrm{i} \varphi_{2}$. These equations are invariant under the infinitesimal gauge transformations, given by

$$
a_{1} \longmapsto \tilde{a}_{1}=a_{1}+\partial_{1} \chi, \quad a_{2} \longmapsto \tilde{a}_{2}=a_{2}+\partial_{2} \chi, \quad \varphi \longmapsto \tilde{\varphi}=\varphi+\mathrm{i} \chi \Phi
$$

where $\chi$ is a real-valued function.

Consider the space of solutions $\left(a_{1}, a_{2}, \varphi_{1}, \varphi_{2}\right)$ of the linearized vortex equations, belonging to the Sobolev space $\left(H^{1}\left(\mathbb{R}^{2} ; \mathbb{R}\right)\right)^{4}$. Note that the infinitesimal gauge transformations with $\chi \in H^{2}\left(\mathbb{R}^{2}\right)$ preserve this space. Following [7], we fix the infinitesimal gauge by imposing on the solutions $\left(a_{1}, a_{2}, \varphi_{1}, \varphi_{2}\right)$ of linearized vortex equations the following orthogonality condition

$$
\partial_{1} a_{1}+\partial_{2} a_{2}+\varphi_{1} \Phi_{2}-\varphi_{2} \Phi_{1}=0 .
$$

It means that $\left(a_{1}, a_{2}, \varphi_{1}, \varphi_{2}\right)$ is $L^{2}$-orthogonal to additive infinitesimal gauge transformation terms $\left(\partial_{1} \chi, \partial_{2} \chi,-\chi \Phi_{2}, \chi \Phi_{1}\right)$ for any $\chi \in H^{2}\left(\mathbb{R}^{2} ; \mathbb{R}\right)$.

We introduce now a linearized vortex operator $\mathcal{D}_{(A, \Phi)}\left(a_{1}, a_{2}, \varphi_{1}, \varphi_{2}\right)$, defined by the left-hand sides of linearized vortex equations and of the orthogonality condition. It is a bounded linear operator, mapping $\left(H^{1}\right)^{4} \rightarrow\left(L^{2}\right)^{4}$. The kernel of $\mathcal{D}_{(A, \Phi)}$ has the (real) dimension $2 N$ (cf. [7]).

We shall identify $\operatorname{ker} \mathcal{D}_{(A, \Phi)}$ with the tangent space of $\mathcal{M}_{N}$ at the point, determined (according to the Taubes theorem) by the collection $\left\{Z_{1}, \ldots, Z_{N}\right\}$ of zeroes of $\Phi$. To do that, consider the symmetric functions $S_{1}, \ldots, S_{N}$ of zeroes of $\Phi$ (equal to the coefficients of the monic polynomial with zeroes at $\left\{Z_{1}, \ldots, Z_{N}\right\}$ ), as coordinates on $\mathcal{M}_{N}$. Fixing the gauge by condition (8), we can consider the components $A_{1}, A_{2}, \Phi$ of vortex solutions as functions of the complex parameters $S_{1}, \ldots, S_{N}$. We obtain solutions of the linearized equations by differentiating these functions with respect to the parameters.

For any $j=1, \ldots, N$ we write $S_{j}$ in the form $S_{j}=S_{j, 1}+\mathrm{i} S_{j, 2}$. We would like to define the kinetic energy metric on $\mathcal{M}_{N}$ in terms of the $L^{2}$-norms of derivatives of $\left(A_{1}, A_{2}, \Phi_{1}, \Phi_{2}\right)$ with respect to $S_{j, k}$-variables with $j=1, \ldots, N, k=1,2$. Unfortunately, these derivatives may be not square integrable. But we can replace $\left(A_{1}, A_{2}, \Phi_{1}, \Phi_{2}\right)$ by suitable gauge equivalent functions, whose derivatives are 
already $L^{2}$-integrable. In other words, we can find gauge factors $\chi_{j, k}$ such that the functions

$$
n_{j, k}=\left(\frac{\partial A_{1}}{\partial S_{j, k}}+\partial_{1} \chi_{j, k}, \frac{\partial A_{2}}{\partial S_{j, k}}+\partial_{2} \chi_{j, k}, \frac{\partial \Phi_{1}}{\partial S_{j, k}}-\chi_{j, k} \Phi_{2}, \frac{\partial \Phi_{2}}{\partial S_{j, k}}+\chi_{j, k} \Phi_{1}\right)
$$

belong to $\left(H^{1}\right)^{4}$ and satisfy the orthogonality condition. We prove also that $\left(S_{1}, \ldots, S_{N}\right) \mapsto n_{j, k}\left(S_{1}, \ldots, S_{N}\right)$ are smooth maps $\mathbb{C}^{N} \rightarrow\left(L^{2}\right)^{4}$ with values in $\left(H^{1}\right)^{4}$. The vectors $n_{j, k}$ with $j=1, \ldots, n, k=1,2$ belong to $\operatorname{ker} \mathcal{D}_{(A, \Phi)}$ and are linearly independent. Hence, they form a basis in $\left.\operatorname{ker} \mathcal{D}_{(A, \Phi)}\right)$. Using this basis, we can identify $T_{(A, \Phi)} \mathcal{M}_{N}$ with $\operatorname{ker} \mathcal{D}_{(A, \Phi)}$.

We can define now the kinetic energy metric $g$ on $\mathcal{M}_{N}$ with the help of $L^{2}$ scalar product. For any $v_{1}, v_{2} \in T_{(A, \Phi)} \mathcal{M}_{N}=\operatorname{kerD}_{(A, \Phi)} \subset\left(H^{1}\right)^{4}$ we set $g\left(v_{1}, v_{2}\right):=\left(v_{1}, v_{2}\right)_{\left(L^{2}\right)^{4}}$. Since the basis vectors $n_{j, k}$ depend smoothly on $S_{1}, \ldots, S_{N}$ (in the sense defined above), the coefficients of the metric, equal to $g_{j, k ; l, m}=\left(n_{j, k}, n_{l, m}\right)_{\left(L^{2}\right)^{4}}$, are smooth functions of $S_{1}, \ldots, S_{N}$. Note that the metric is invariant under simultaneous translations, rotations and complex conjugation of all positions of vortices.

To establish that all the objects above are well-defined, we should prove the following theorem.

Theorem 2. Let $A_{1}, A_{2}, \Phi$ are the components of vortex solutions. Then

1. $A_{1}, A_{2}, \Phi$ depend smoothly on $S_{1}, \ldots, S_{N}$ in the sense that for any fixed $z_{0}$ the function $\Phi\left(z_{0} ; S_{1}, \ldots, S_{N}\right)$ is a smooth function of $S_{1}, \ldots, S_{N}$ and the same for $A_{1}, A_{2}$.

2. We can choose $\chi_{j, k}$ such that the $n_{j, k}$, defined by the formula (12), belong to $\left(H^{1}\right)^{4}$ and satisfy the orthogonality condition.

3. The maps $\left(S_{1}, \ldots, S_{N}\right)$ are smooth maps of $\mathbb{C}^{N}$ to $\left(L^{2}\right)^{4}$ with values in $\left(H^{1}\right)^{4}$.

4. The vectors $n_{j, k}$ for $j=1, \ldots, N$ and $k=1,2$ are linearly independent.

Some remarks on the proof of this theorem will be given in Section 5.

\section{Dynamical Problem}

The dynamical $(2+1)$-dimensional Higgs model is given by the action functional

$$
\begin{aligned}
\mathcal{S}(A, \Phi)= & \frac{1}{2} \int_{t_{1}}^{t_{2}} \mathrm{~d} t \int_{\mathbb{R}^{2}}\left\{\left(\left|\left(\partial_{0}-\mathrm{i} A_{0}\right) \Phi\right|^{2}+F_{01}^{2}+F_{02}^{2}\right)\right. \\
& \left.-\left(\left|\left(\partial_{1}-\mathrm{i} A_{1}\right) \Phi\right|^{2}+\left|\left(\partial_{2}-\mathrm{i} A_{2}\right) \Phi\right|^{2}+F_{12}^{2}+\frac{1}{4}\left(|\Phi|^{2}-1\right)^{2}\right)\right\} \mathrm{d} x \mathrm{~d} y .
\end{aligned}
$$


Here $\Phi(t, x, y)$ is a smooth complex-valued function, $A_{j}(t, x, y)$ are smooth realvalued functions $(j=0,1,2) ; F_{j k}:=\partial_{j} A_{k}-\partial_{k} A_{j}$ and we have used the notation $\partial_{0}=\partial_{t}, \partial_{1}=\partial_{x}$, and $\partial_{2}=\partial_{y}$.

The action functional can be represented in the standard form

$$
\mathcal{S}=\int(T-V) \mathrm{d} t
$$

where the potential energy $V$ is given by the formula (1) and the kinetic energy $T$ is equal to

$$
T=\frac{1}{2} \int_{\mathbb{R}^{2}}\left(\left|\left(\partial_{0}-\mathrm{i} A_{0}\right) \Phi\right|^{2}+F_{01}^{2}+F_{02}^{2}\right) \mathrm{d} x \mathrm{~d} y .
$$

We would like to describe the moduli space of solutions of the corresponding dynamical problem

$$
\delta \mathcal{S}(A, \Phi)=0 .
$$

But, in contrast with vortices (which are the static solutions of our dynamical problem), we have little hope to obtain an explicit description of this moduli space. However, following an idea, proposed in [7] and [3], we can consider geodesics on $\mathcal{M}_{N}$ in the kinetic energy metric as a good approximation to the trajectories of $N$ slowly moving vortices. We use this approximation in order to describe the scattering of $N$ vortices after their symmetric head-on collision.

Unfortunately, there is no explicit formula for the kinetic energy metric. We use instead the invariance of this metric under rotations and complex conjugation to deduce a qualitative description of the scattering. Namely, we prove that the trajectories of vortices are rotated by the angle $\pi / N$ after the collision. In particular, the head-on collision of two vortices results in the rotation of their trajectories by the angle $\pi / 2$, so in this case we have the right-angle scattering. This latter result was already obtained in a number of papers (see [6] and also [1,4,5]).

To obtain our result on scattering, we consider a geodesic on $\mathcal{M}_{N}$ passing through the origin $S_{1}=\cdots=S_{N}=0$ with the tangent vector $\vec{v}=\left(0,0, \ldots, 0,(-1)^{N+1}\right)$ at this point. (The sign $(-1)^{N}$ in the formula for $\vec{v}$ is due to the equality $S_{N}=$ $\left(-Z_{1}\right)\left(-Z_{2}\right) \ldots\left(-Z_{N}\right)=(-1)^{N} Z_{1} Z_{2} \ldots Z_{N}$.) Suppose that our geodesic is parameterized by: $S_{1}=S_{1}(s), \ldots, S_{N}=S_{N}(s)$. (Here $s$ is a natural parameter on the geodesic.) Let the origin correspond to $s=0$. The rotation of all zeroes by the angle $2 \pi / N$ (that is, $Z_{k}^{\prime}=\mathrm{e}^{2 \pi \mathrm{i} / N} Z_{k}$ ) does not change the vector $\vec{v}$ and the complex conjugation $\left(Z_{k}^{\prime}=\bar{Z}_{k}\right)$ does not change it too. Since the metric is invariant under these transformations, our geodesics is mapped to a geodesic with the same tangent vector, and since the metric is smooth, our geodesic must be 
mapped to itself. Hence $S_{k}(s)=\mathrm{e}^{2 \pi \mathrm{i} k / N} S_{k}(s)$ and $S_{k}(s)=\overline{S_{k}(s)}$ and therefore $S_{1}(s)=\cdots=S_{N-1}(s)=0, S_{N}(s)$ is real. Denote $(-1)^{N} S_{N}(s)$ by $\lambda(s)$. It is evident that $\lambda(0)=0$ and $\lambda(s)$ decreases and changes its sign from the positive to negative, when $s$ passes the origin.

In terms of the original coordinates $\left(Z_{1}, \ldots, Z_{N}\right)$ our geodesic (which is smooth in symmetric coordinates) corresponds to the following motion of $N$ vortices. For $\lambda(s)>0$ (i.e., $s<0$ ) we have $N$ trajectories, described by

$$
s \longmapsto\left(\sqrt[N]{\lambda(s)}, \sqrt[N]{\lambda(s)} \mathrm{e}^{2 \pi \mathrm{i} / N}, \ldots, \sqrt[N]{\lambda(s)} \mathrm{e}^{2 \pi \mathrm{i}(N-1) / N}\right)
$$

while for $\lambda(s)<0$ (i.e., $s>0$ ) we have $N$ trajectories, rotated by the angle $\pi / N$

$$
s \longmapsto\left(\sqrt[N]{|\lambda(s)|} \mathrm{e}^{\pi \mathrm{i} / N}, \sqrt[N]{|\lambda(s)|} \mathrm{e}^{3 \pi \mathrm{i} / N}, \ldots, \sqrt[N]{|\lambda(s)|} \mathrm{e}^{(2 N-1) \pi \mathrm{i} / N}\right)
$$

In other words, our geodesic describes the scattering process of the following type. Before the collision $N$ vortices are moving to the origin along the rays: $\arg z=0$, $\arg z=2 \pi / N, \ldots, \arg z=2(N-1) \pi / N$. Then they collide at the origin and after that move away along the rays $\arg z=\pi / N, \ldots, \arg z=(2(N-1)+1) \pi / N$.

\section{Remarks on Proof of Theorem 2}

Let the vortex solution have the form (8). Denote $p(z):=\left(z-Z_{1}\right) \ldots\left(z-Z_{N}\right)$, so that $\Phi(z)=p(z) f(z)$. Then for the function $w:=2 \ln f+\ln \left(1+|p|^{2}\right)$ we obtain the equation

$$
\Delta w=\frac{|p|^{2}}{1+|p|^{2}} \mathrm{e}^{w}-1+\Delta \ln \left(1+|p|^{2}\right) .
$$

Let $S$ denote $\left(S_{1}, \ldots, S_{N}\right)$. In Chapter 3 of [2] it was proved that for every collection $\left\{Z_{1}, \ldots, Z_{N}\right\}$ we have a unique solution of (19) in Sobolev space $H^{2}\left(\mathbb{R}^{2}\right)$. We denote it by $w(S)$. We shall prove that $w(S)$ depend smoothly on $S$ with the help of the implicit function theorem.

It is not so hard to prove that the map $F: H^{2}\left(\mathbb{R}^{2}\right) \times \mathbb{C}^{N} \rightarrow L^{2}$, given by

$$
F(v ; S)=-\Delta v+\frac{|p(S)|^{2}}{1+|p(S)|^{2}} \mathrm{e}^{v}-1+\Delta \ln \left(1+|p(S)|^{2}\right)
$$

is smooth and in order to apply the implicit function theorem for Banach spaces, we should show that the linear operator $F_{v}^{\prime} h: H^{2}\left(\mathbb{R}^{2}\right) \rightarrow L^{2}\left(\mathbb{R}^{2}\right)$, namely, $F_{v}^{\prime} h=$ 
$-\Delta h+\frac{|p(S)|^{2}}{1+|p(S)|^{2}} \mathrm{e}^{v} h$, is invertible for $v=w(S)$, i.e., that $A: h \rightarrow-\Delta h+$ $|\Phi|^{2} h$ is invertible.

Choose a smooth non-negative function $\epsilon$, vanishing outside some ball $B_{R}=$ $B(0, R)$, such that $\delta \leq|\Phi|^{2}+\epsilon \leq 1$ for some $\delta>0$. Then the operator $A_{\epsilon}$ defined by $A_{\epsilon} h=-\Delta h+\left(|\Phi|^{2}+\epsilon\right) h$ is invertible. Indeed, $\left\|A_{\epsilon} h\right\|_{L^{2}} \geq \gamma\|h\|_{H^{2}}$ for some $\gamma>0$, because

$$
\left\|\left(1+x^{2}+y^{2}\right) \hat{h}\right\|_{L^{2}} \leq \mathrm{const}\left\|A_{\epsilon} h\right\|_{L^{2}}
$$

where $\hat{h}=\mathcal{F} h$ denotes the Fourier transform.

Now note that $A_{\epsilon} h=A h+\epsilon h \Longleftrightarrow h=A_{\epsilon}^{-1} A h+A_{\epsilon}^{-1}(\epsilon h)$, implying that $A_{\epsilon}^{-1} A h=h-A_{\epsilon}^{-1}(\epsilon h)=\left(\mathrm{Id}-K_{\epsilon}\right) h$, where Id is the identity operator and $K_{\epsilon} h=A_{\epsilon}^{-1}(\epsilon h)$ is a compact operator, since the embedding $H^{2}\left(B_{R}\right) \hookrightarrow L^{2}\left(B_{R}\right)$ is compact. If $\left(\mathrm{Id}-K_{\epsilon}\right) h=0$, then $A h=A_{\epsilon}\left(\mathrm{Id}-K_{\epsilon}\right) h=0$ and so $-\Delta h+$ $|\Phi|^{2} h=0$. But this implies $h=0$, since $|\Phi| \rightarrow 1$ for $|z| \rightarrow \infty$. The Fredholm theorem implies now that $\mathrm{Id}-K_{\epsilon}$ is invertible, and therefore the operator $A=$ $A_{\epsilon} \circ\left(\mathrm{Id}-K_{\epsilon}\right)$ is also invertible.

So we can apply the implicit function theorem and prove that $w$ depends smoothly on $S_{1}, \ldots, S_{N}$ in $H^{2}\left(\mathbb{R}^{2}\right)$. But the $H^{2}$-norm majorizes the uniform norm, so $w$ depends smoothly of $S_{1}, \ldots, S_{N}$ also in the sense of Section 3. Since $\Phi, A_{1}$, $A_{2}$ can be expressed through $w$, they also depend smoothly on $S_{1}, \ldots, S_{N}$ in the sense of Section 3.

Let us now determine $\chi_{j, k}$. Substituting the expressions (12) into the orthogonality condition, we obtain the equation $-\Delta \chi_{j, k}+|\Phi|^{2} \chi_{j, k}=-|\Phi|^{2} \frac{\partial \vartheta}{\partial S_{j, k}}$, where $\vartheta=\arg \Phi$. Choose a smooth function $\rho(z ; S)$ satisfying the following conditions: $0 \leq \rho \leq 1, \rho(\cdot ; S)$ is compactly supported, $\rho(z ; S) \equiv 1$ in some ball containing all zeroes of $\Phi(z ; S)$, and $\rho$ depends smoothly on $S$. Then for $v_{j, k}:=\chi_{j, k}+(1-\rho) \frac{\partial \vartheta}{\partial S_{j, k}}$ we obtain the equation

$$
-\Delta v_{j, k}+|\Phi|^{2} v_{j, k}=-\rho|\Phi|^{2} \frac{\partial \vartheta}{\partial S_{j, k}}-\Delta\left((1-\rho) \frac{\partial \vartheta}{\partial S_{j, k}}\right) .
$$

The function in the right hand side of this equation is smooth and has a compact support, so we can apply our result on $-\Delta+|\Phi|^{2}$ and then use regularity theory for elliptic equations to show that $v_{j, k}$ are smooth. We can show that $v_{j, k}$ depend smoothly on $S_{k}$ in the $H^{2}$ norm using the same argument as for $w$. Finally we determine $\chi_{j, k}=v_{j, k}-(1-\rho) \frac{\partial \vartheta}{\partial S_{j, k}}$. 


\section{Acknowledgements}

While preparing this paper, the author was partly supported by the RFBR grant 05-01-00498 and by the program of Support of Scientific Schools (grant NSH6705.2006.1).

\section{References}

[1] Abdelwahid F. and Burzlaff J., Existence Theorem for $90^{\circ}$ Vortex-Vortex Scattering, J. Math. Phys. 35 (1994) 4651-4660.

[2] Jaffe A. and Taubes C., Vortices and Monopoles, Birkhäuser, Boston, 1980.

[3] Manton N., A Remark on the Scattering of BPS Monopoles, Phys. Lett. 110B (1982) 54-56.

[4] Ruback P., Vortex String Motion in the Abelian Higgs Model, Nucl. Phys. B296 (1988) 669-678.

[5] Samols T., Vortex Scattering, Comm. Math. Phys. 145 (1992) 149-179.

[6] Sergeev A. and Chechin S., Scattering of Slowly Moving Vortices in the Abelian (2+1)-dimensional Higgs Model, Theor. Math. Phys. 85 (1990) 1289-1299.

[7] Stuart D., Dynamics of Abelian Higgs Vortices in the Near Bogomolny Regime, Comm. Math. Phys. 159 (1994) 51-91.

Roman Palvelev

Steklov Mathematical Institute

Gubkina 8, 119991 Moscow

RUSSIA

E-mail address: palvelev@front.ru 